\title{
Species diversity and abundance of Euphylliidae (Cnidaria: Anthozoa: Scleractinia) corals in Tioman, Redang and Payar Islands, Peninsular Malaysia
}

\author{
Shahbudin Saad* and Fikri Akmal Khodzori \\ Department of Marine Science, Kulliyyah of Science, International Islamic University Malaysia, Jalan Sultan Ahmad \\ Shah, Bandar Indera Mahkota, 25200 Kuantan, Pahang, Malaysia
}

${ }^{*}$ Corresponding author: ocean@iium.edu.my

\begin{abstract}
The number of endangered coral species is increasing over the past decades due to multiple stresses and threats. Euphylliidae corals are among the species heavily targeted for the marine aquarium trade due to their colourful appearance and aesthetic importance. However, their distribution in Peninsular Malaysia has not been thoroughly investigated. Present study aims to investigate the diversity and abundance patterns of euphylliid species at 36 reef sites in Marine Protected Areas of Tioman, Redang and Payar Islands. Video transect surveyed a total of 671 euphylliids individuals belonging to six species from three genera. The diversity and evenness indices of euphylliids were significantly higher $(\mathrm{P}<0.05)$ in Tioman compared to Redang and Payar Islands. Based on the total number of euphylliids recorded in the study areas, Euphyllia ancora (29.5\%) was found to be the most dominant in Tioman, Plerogyra sinuosa (92.3\%) in Redang and Physogyra lichtensteini (98.0\%) in Payar. Current findings suggest that the combined factors of habitat heterogeneity, especially the reef morphology and substratum condition, and anthropogenic impacts have most likely influenced the distribution of this endangered coral species in Peninsular Malaysia.
\end{abstract}

Keywords: Euphylliidae corals, Diversity, Abundance pattern, Endangered species, Coral video transect, Peninsular Malaysia

\section{Introduction}

The Indo-Pacific coral family Euphylliidae Alloiteau, 1952 formerly known as the Euphyllidae Veron, 2000 contains five genera, namely Euphyllia, Catalaphyllia, Nemenzophyllia, Plerogyra and Physogyra (Veron, 2000). Previously, these genera have been classified based on morphological characteristics of solid, smooth and widely spaced septa with expanded tentacles, vesicles or mantles during the daytime (Veron, 2000). Recent phylogenetic relationships in accordance with evidence by fleshy vesicle mantle formations reveal that Plerogyra, Physogyra and Nemenzophyllia belong to Incertae sedis, that is closely related to Blastomussa, while only Euphyllia and Catalaphyllia are part of the Euphylliidae family (Fukami et al., 2008; Kitahara et al., 2010; Benzoni et al., 2014; Khodzori et al., 2017). However, this paper describes the Euphylliidae using previous taxonomic classification of Scleractinia following Veron (2000).

The Euphylliidae corals are among the most beautiful reef building corals due to their brightly coloured fleshy polyps (Veron, 2000). Moreover, their colourful appearance and fast-growth rate are the encouraging factors for these species to be exploited for the marine aquarium trade (Wabnitz et al., 2003; Rhyne et al., 2014). Previous study showed that more than $75 \%$ of the Euphyllia spp., particularly E. ancora and E. glabrescens were exported to the United State since 2009 until 2011 (Rhyne et al., 2014). Majority of them have been exported from countries within the Coral Triangle region, with Indonesia as the world's largest exporter (Tissot et al., 2010; Rhyne et al., 2014).
Wild harvesting and exploitation of marine ornamental species including the live corals, coral reef fish and invertebrates may result in reducing the population size of susceptible species, in particular the rare and endangered species (Wilkinson, 2008; Carpenter et al., 2008; Tissot et al., 2010 ). It has been estimated that $36 \%$ of coral reefs will be degraded in the next 30 years if the marine wild populations are continuously exploited in unsustainable ways together with persistent natural phenomena and other human induced disturbances (Tissot et al., 2010; Thornhill, 2012; Rhyne et al., 2014). As for conservation action for the Euphylliidae corals, the International Union for Conservation of Nature and Natural Resources (IUCN) has declared that most of the Euphylliidae corals are near-threatened and vulnerable species. Nevertheless, some of them are yet to be assessed due to deficiency of data.

Species lists of hard corals including the Euphylliidae have previously been published by Harborne et al. (2000) at Marine Protected Areas (MPAs) of Tioman and Redang. Five euphylliids (Physogyra lichtensteini, Euphyllia ancora, E. divisa, E. glabrescens, E. yaeyamaensis and Plerogyra sinuosa) have been recorded in Tioman and four of them (P. lichtensteini, E, divisa, E. glabrescens, and P. sinuosa) in Redang (Harborne et al., 2000). Recent study showed one additional species of $E$. cristata, that raises the species count to six euphylliids in Tioman (Khodzori et al., 2015). However, limited data and information on species richness patterns of the Euphylliidae have been documented in Peninsular Malaysia as compared to Sabah where a good deal of information has been published (e.g. Waheed and Hoeksema, 2013; Waheed and Hoeksema, 2014; Waheed et al., 2015). It is important to update the species list of these 
near-threatened and vulnerable species in order to perform a continuous evaluation of their current status in the MPAs. Therefore, the present study contributes to filling the gap in information regarding the percentage coverage and abundance patterns as well as updating the species list of the Euphylliidae corals in Tioman, Redang and Payar Islands.

\section{Materials and Methods}

\section{Study Area}

Fieldwork was conducted during September 2013 - February 2014 in Tioman, Redang and Payar Islands (Figure 1 and Table 1). Tioman and Redang Islands were divided into 13 (T1 - T13) and 16 (R1 -R16) sampling sites, respectively. Payar Island which is smaller in size compared to Tioman and Redand was divided into 7n (P1 - P7) sampling sites (Table 1). These islands are recognized as popular tourism destinations in Peninsular Malaysia. According to the recent data, an average of 793, 359 tourists have visited the marine parks, including these islands (DMPM, 2013).

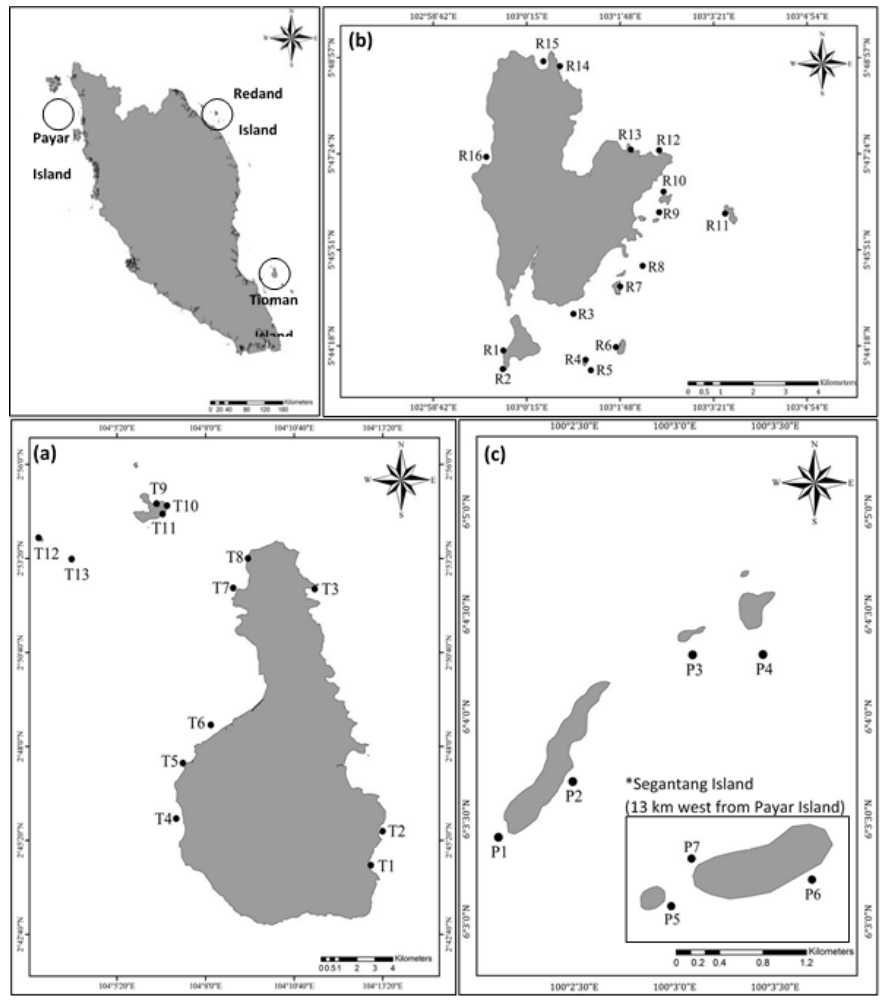

Figure 1: Study areas in Peninsular Malaysia (a) 13 reef sites in Tioman Island, (b) 16 reef sites in Redang Island, and (c) 7 reef sites in Payar Island

\section{Field Sampling}

A field survey was carried out at 36 reef sites using the Coral Video Transect (CVT) technique as adopted by Liew et al. (2012) with some adaptation. A total of 144 transects were used throughout the sampling period. An underwater camera (Olympus TG-3 protected with Olympus PT-053 waterproof case) was used to record video of benthic communities along the transect length with approximately $50 \mathrm{~cm}$ distance from the substrate and at a perpendicular angle to the bottom. The camera was run along the transect lines at a speed of 5 meter per minute to record sharp and clear images. The additional pictures of living Euphylliidae corals surrounding reef sites were photographed in situ at both actual size and macro shots including the condition when the tentacles or vesicles were inflated and deflated to facilitate in the identification.

Table 1. Locality data of reef sites and maximum depth of transect in Tioman, Redang and Payar Islands

\begin{tabular}{|c|c|c|c|c|c|}
\hline $\begin{array}{l}\text { Survey } \\
\text { Site }\end{array}$ & Island & Reef Site & $\begin{array}{l}\text { Latitude } \\
\text { (N) }\end{array}$ & $\begin{array}{l}\text { Longitude } \\
\text { (E) }\end{array}$ & $\begin{array}{l}\text { Max. } \\
\text { Transect } \\
\text { Depth (m) }\end{array}$ \\
\hline T1 & \multirow{13}{*}{$\begin{array}{l}\text { Tioman } \\
\text { Island }\end{array}$} & Sanggit Bay & $02^{\circ} 44^{\prime} 37.9^{\prime \prime}$ & $104^{\circ} 12^{\prime} 58.4^{\prime \prime}$ & 10 \\
\hline $\mathrm{T} 2$ & & Benuang Bay & $02^{\circ} 45^{\prime} 35.7^{\prime \prime}$ & $104^{\circ} 13^{\prime} 19.7^{\prime \prime}$ & 12 \\
\hline $\mathrm{T} 3$ & & Dalam Bay & $02^{\circ} 52^{\prime} 28.3^{\prime \prime}$ & $104^{\circ} 11^{\prime} 16.9^{\prime \prime}$ & 8 \\
\hline $\mathrm{T} 4$ & & $\begin{array}{l}\text { Genting } \\
\text { Village }\end{array}$ & $02^{\circ} 45^{\prime} 57.3^{\prime \prime}$ & $104^{\circ} 07^{\prime} 06.9^{\prime \prime}$ & 10 \\
\hline T5 & & Tomok Island & $02^{\circ} 47^{\prime} 31.6^{\prime \prime}$ & $104^{\circ} 07^{\prime} 18.9^{\prime \prime}$ & 9 \\
\hline $\mathrm{T} 6$ & & $\begin{array}{l}\text { Renggis } \\
\text { Island }\end{array}$ & $02^{\circ} 48^{\prime} 36.6^{\prime \prime}$ & $104^{\circ} 08^{\prime} 09.4^{\prime \prime}$ & 12 \\
\hline $\mathrm{T} 7$ & & Soyak Island & $02^{\circ} 52 ' 29.7^{\prime \prime}$ & $104^{\circ} 08^{\prime} 49.9^{\prime \prime}$ & 8 \\
\hline $\mathrm{T} 8$ & & Terdau Bay & $02^{\circ} 53^{\prime} 20.0^{\prime \prime}$ & $104^{\circ} 09^{\prime} 16.6^{\prime \prime}$ & 10 \\
\hline T9 & & Gado Bay & $02^{\circ} 54^{\prime} 53.4^{\prime \prime}$ & $104^{\circ} 06^{\prime} 31.4^{\prime \prime}$ & 12 \\
\hline $\mathrm{T} 10$ & & Tulai Bay & $02^{\circ} 54^{\prime} 49.9^{\prime \prime}$ & $104^{\circ} 06^{\prime} 50.3^{\prime \prime}$ & 15 \\
\hline T11 & & Bayan Bay & $02^{\circ} 54^{\prime} 35.6^{\prime \prime}$ & $104^{\circ} 06^{\prime} 42.4^{\prime \prime}$ & 12 \\
\hline T12 & & Sepoi Island & $02^{\circ} 53^{\prime} 55.3^{\prime \prime}$ & $104^{\circ} 02^{\prime} 58.3^{\prime \prime}$ & 16 \\
\hline $\mathrm{T} 13$ & & Labas Island & $02^{\circ} 53^{\prime} 19.1^{\prime \prime}$ & $104^{\circ} 03^{\prime} 58.1^{\prime \prime}$ & 18 \\
\hline R1 & \multirow{16}{*}{$\begin{array}{l}\text { Redang } \\
\text { Island }\end{array}$} & Tg. Ara & $05^{\circ} 44^{\prime} 13.6^{\prime \prime}$ & $102^{\circ} 59^{\prime} 51.9^{\prime \prime}$ & 8 \\
\hline R2 & & Terumbu Kili & $05^{\circ} 43^{\prime} 55.7^{\prime \prime}$ & $102^{\circ} 59^{\prime} 51.3^{\prime \prime}$ & 12 \\
\hline R3 & & $\begin{array}{l}\text { Tg. Cina } \\
\text { Terjun }\end{array}$ & $05^{\circ} 46^{\prime} 26.1^{\prime \prime}$ & $103^{\circ} 01^{\prime} 13.2^{\prime \prime}$ & 14 \\
\hline R4 & & Batu Ling & $05^{\circ} 44^{\prime} 11.9^{\prime \prime}$ & $103^{\circ} 01^{\prime} 10.1^{\prime \prime}$ & 14 \\
\hline R5 & & $\begin{array}{l}\text { Chupak } \\
\text { Island }\end{array}$ & $05^{\circ} 44^{\prime} 04.6^{\prime \prime}$ & $103^{\circ} 01^{\prime} 13.7^{\prime \prime}$ & 12 \\
\hline R6 & & $\begin{array}{l}\text { Ekor Tebu } \\
\text { Island }\end{array}$ & $05^{\circ} 44^{\prime} 16.8^{\prime \prime}$ & $103^{\circ} 01^{\prime} 43.9^{\prime \prime}$ & 8 \\
\hline R7 & & $\begin{array}{l}\text { Kerengga } \\
\text { Besar Island }\end{array}$ & $05^{\circ} 45^{\prime} 15.5^{\prime \prime}$ & $103^{\circ} 01^{\prime} 48.1^{\prime \prime}$ & 12 \\
\hline R8 & & Chek Isa & $05^{\circ} 45^{\prime} 35.4^{\prime \prime}$ & $103^{\circ} 02^{\prime} 10.4^{\prime \prime}$ & 12 \\
\hline R9 & & Mak Chantek & $05^{\circ} 46^{\prime} 27.3^{\prime \prime}$ & $103^{\circ} 02^{\prime} 26.9^{\prime \prime}$ & 10 \\
\hline R10 & & $\begin{array}{l}\text { Paku Besar } \\
\text { Island }\end{array}$ & $05^{\circ} 46^{\prime} 47.3^{\prime \prime}$ & $103^{\circ} 02^{\prime} 31.3^{\prime \prime}$ & 8 \\
\hline R11 & & Lima Island & $05^{\circ} 46^{\prime} 26.1^{\prime \prime}$ & $103^{\circ} 03^{\prime} 32.5^{\prime \prime}$ & 15 \\
\hline R12 & & $\begin{array}{l}\text { Tg. Gua } \\
\text { Kawah }\end{array}$ & $05^{\circ} 47^{\prime} 27.3^{\prime \prime}$ & $103^{\circ} 02^{\prime} 26.9^{\prime \prime}$ & 12 \\
\hline R13 & & $\begin{array}{l}\text { Mak Delah } \\
\text { Bay }\end{array}$ & $05^{\circ} 47^{\prime} 27.9^{\prime \prime}$ & $103^{\circ} 01^{\prime} 58.7^{\prime \prime}$ & 10 \\
\hline R14 & & Tg. Tok Kong & $05^{\circ} 48^{\prime} 48.7^{\prime \prime}$ & $103^{\circ} 00^{\prime} 48.1^{\prime \prime}$ & 12 \\
\hline R15 & & $\begin{array}{l}\text { Tg. Chagar } \\
\text { Hutang }\end{array}$ & $05^{\circ} 48^{\prime} 53.4^{\prime \prime}$ & $103^{\circ} 00^{\prime} 31.7^{\prime \prime}$ & 18 \\
\hline R16 & & $\begin{array}{l}\text { Pasir Mak } \\
\text { Simpan }\end{array}$ & $05^{\circ} 47^{\prime} 20.9^{\prime \prime}$ & $102^{\circ} 59^{\prime} 34.9^{\prime \prime}$ & 10 \\
\hline P1 & \multirow{7}{*}{$\begin{array}{l}\text { Payar } \\
\text { Island }\end{array}$} & Banana Reef & $06^{\circ} 03^{\prime} 41.0^{\prime \prime}$ & $100^{\circ} 02^{\prime} 29.6^{\prime \prime}$ & 8 \\
\hline $\mathrm{P} 2$ & & Coral Garden & $06^{\circ} 03^{\prime} 24.7^{\prime \prime}$ & $100^{\circ} 02^{\prime} 07.4^{\prime \prime}$ & 12 \\
\hline P3 & & Kaca Island & $06^{\circ} 04^{\prime} 18.1^{\prime \prime}$ & $100^{\circ} 03^{\prime} 05.4^{\prime \prime}$ & 10 \\
\hline P4 & & Lembu Island & $06^{\circ} 04^{\prime} 18.2^{\prime \prime}$ & $100^{\circ} 03^{\prime} 26.4^{\prime \prime}$ & 8 \\
\hline P5 & & $\begin{array}{l}\text { Segantang } \\
\text { Bay }\end{array}$ & $06^{\circ} 02^{\prime} 35.8^{\prime \prime}$ & $099^{\circ} 55^{\prime} 27.5^{\prime \prime}$ & 18 \\
\hline P6 & & $\begin{array}{l}\text { Anemone } \\
\text { Garden }\end{array}$ & $06^{\circ} 02^{\prime} 38.0^{\prime \prime}$ & $099^{\circ} 55^{\prime} 26.8^{\prime \prime}$ & 15 \\
\hline P7 & & Tyre Reef & $06^{\circ} 02^{\prime} 39.7^{\prime \prime}$ & $099^{\circ} 55^{\prime} 31.6 "$ & 18 \\
\hline
\end{tabular}

\section{Image Processing and Data Analysis}

All recorded videos of benthic communities for every reef site were converted to 300 snapshot images (75 images per transect tape) using the video converter. Total numbers of euphylliids were quantified into average percentage coverage for each species recorded at every island. The abundance of 
each Euphylliidae species for every reef site was determined based on the total colony number counted in the snapshot images. It was categorized based on a rating scale of one to five dots: $\bullet=$ one or few $(1-5$ colonies $), \bullet=$ uncommon $(6-$ 10 colonies $), \cdots=\operatorname{common}(11-20$ colonies $), \cdots \bullet=$ abundant (21 - 30 colonies) and $\bullet \bullet \bullet:$ dominant (> 30 colonies). The Shannon-Weiner diversity index, H' (Shannon and Weaver, 1949) and Pielou evenness index, J' (Pielou, 1966) were used to determine the diversity and evenness of the Euphylliidae corals recorded for every reef site. This univariate analysis was done using PAST (Paleontological Statistic) software version 3 (Hammer et al., 2001). The statistical comparison of One-Way Analysis of Variance (ANOVA) was performed to determine the significance of differences $(p<0.05)$ in the diversity and evenness value of the Euphylliidae among reefs sites and study areas using Minitab 17 software.

\section{Results}

\section{Species Checklist of Euphylliidae Corals}

A total of six species and three genera of Euphyllia, Plerogyra and Physogyra were identified in the study area following the book of Corals of the World (Veron, 2000) (Table 2, Figure 2). Based on the previous study done by Harborne et al. (2000) and compilation study by Affendi and Rosman (2012), species E. cristata was not documented in Tioman and Redang Islands, while species P. lichtensteini and E. glabrescens were not recorded in Payar Island. These species can be added to the existing list of coral species found in these islands. Other species recorded during earlier studies, but not encountered during the present survey are E. yaeyamaensis (Veron and Hudgson, 1989), E. paradivisa (Veron and Fenner, 2000), E. paraglabrescens (Veron and Fenner, 2000) and Catalaphyllia jardinei (Veron and Hudgson, 1989).

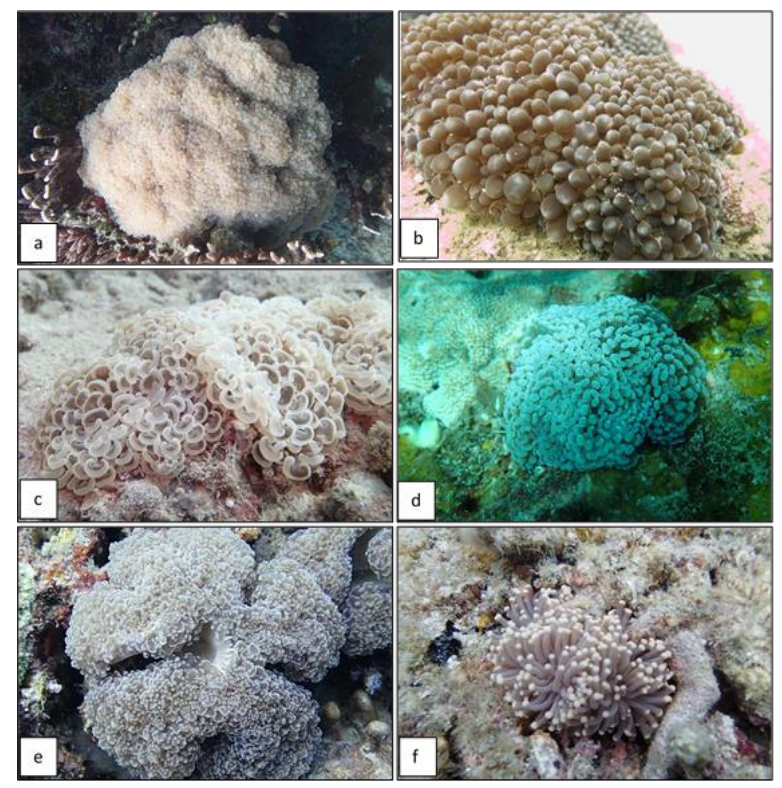

Figure 2: Euphylliidae corals recorded in Tioman, Redang and Payar Islands. (a) P. sinuosa, (b) P. lichtensteini, (c) E. ancora, (d) E. cristata, (e) E. divisa, (f) E.glabrescens.

\section{Current Status of Euphylliidae Corals}

Overall, a total of 671 individuals of euphylliids belonging to six species from three genera, Plerogyra, Physogyra and Euphyllia, were recorded in the study area. All six species were recorded in Tioman Island. E. ancora showed the highest average coverage $(29.5 \% \pm 1.5)$ followed by $E$. divisa $(19.7 \% \pm 1.3)$, P. sinuosa $(19.1 \% \pm 0.7)$ and E. cristata $(17.9 \%$ \pm 1.0 ). P. lichtensteini and E. glabrescens showed the lowest average coverage, covering the same percentage $(6.9 \% \pm 0.4)$. Among these species, E. ancora was dominant while E. divisa and E. cristata, were common species of euphylliids at $\mathrm{T} 2$ and no euphylliids were recorded at T4. P. lichtensteini and E. glabrescens were only found in a few colonies at certain reef sites in Tioman (Table 3).

In Redang Island, a total of five species from two genera (Plerogyra and Euphyllia) were recorded. P. sinuosa showed the highest average coverage with $92.3 \% \pm 4.2$ and was found to be the dominant species of euphylliids at four (R4, R8, R14 and R16) reef sites. E. cristata and E. ancora showed the lowest average coverage $(0.3 \% \pm 0.1)$ and were found only at R1 and R4, respectively. All Euphyllia spp. (E. cristata, E. ancora, E. divisa and E. glabrescens) were found in a few colonies at certain reef sites in Redang (Table 4). In Payar, less number of the Euphylliidae corals was recorded with only two species, namely $P$. lichtensteini and $E$. glabrescens. $P$. lichtensteini showed the highest average coverage $(98.0 \% \pm 6.7)$. It was found at all the reef sites and was categorized as the dominant species of euphylliids at P1 and P2. Out of 7 reef sites. E. glabrescens occurred at P1 and $\mathrm{P} 2$, in a few colonies in Payar (Table 5).

Table 2. Checklist of the Euphylliidae from 36 reef sites in Tioman, Redang and Payar Islands. The number of sites indicates the species occurrence frequencies. Previous records are indicated as follows: $\mathrm{a}=$ Harborne et al. (2000),

$\mathrm{b}=$ Affendi and Rosman (2012), cross (-) = unrecorded species from the previous studies

\begin{tabular}{|c|c|c|c|c|}
\hline $\begin{array}{l}\text { No. of } \\
\text { Species }\end{array}$ & $\begin{array}{l}\text { Eupylliidae Species/ Current } \\
\text { Records }\end{array}$ & $\begin{array}{l}\text { No. of } \\
\text { Sites }\end{array}$ & $\begin{array}{l}\text { Previous } \\
\text { Records }\end{array}$ & $\begin{array}{l}{ }^{*} \text { IUCN } \\
\text { Status }\end{array}$ \\
\hline & Tioman Island & & & \\
\hline 1 & Plerogyra sinuosa (Dana, 1846) & 9 & $\mathrm{a}, \mathrm{b}$ & NT \\
\hline 2 & $\begin{array}{l}\text { Physogyra lichtensteini (Milne } \\
\text { Edwards and Haime, 1851) }\end{array}$ & 6 & $\mathrm{~b}$ & $\mathrm{~V}$ \\
\hline 3 & $\begin{array}{l}\text { Euphyllia ancora (Veron and } \\
\text { Pichon, 1980) }\end{array}$ & 7 & $\mathrm{a}, \mathrm{b}$ & V \\
\hline 4 & Euphyllia cristata (Chevalier, 1971) & 7 & - & V \\
\hline 5 & $\begin{array}{l}\text { Euphyllia divisa (Veron and Pichon, } \\
1980 \text { ) }\end{array}$ & 6 & $\mathrm{a}, \mathrm{b}$ & NT \\
\hline 6 & $\begin{array}{l}\text { Euphyllia glabrescens (Chamisso } \\
\text { and Eysenhardt, 1821) } \\
\text { Redang Island }\end{array}$ & 5 & $a, b$ & NT \\
\hline 1 & Plerogyra sinuosa (Dana, 1846) & 15 & $a, b$ & NT \\
\hline 2 & $\begin{array}{l}\text { Euphyllia ancora (Veron and } \\
\text { Pichon, 1980) }\end{array}$ & 1 & $\mathrm{~b}$ & V \\
\hline 3 & Euphyllia cristata (Chevalier, 1971) & 1 & - & V \\
\hline 4 & $\begin{array}{l}\text { Euphyllia divisa (Veron and Pichon, } \\
1980 \text { ) }\end{array}$ & 8 & $a, b$ & NT \\
\hline 5 & $\begin{array}{l}\text { Euphyllia glabrescens (Chamisso } \\
\text { and Eysenhardt, 1821) } \\
\text { Payar Island }\end{array}$ & 2 & $\mathrm{a}, \mathrm{b}$ & NT \\
\hline 1 & $\begin{array}{l}\text { Physogyra lichtensteini (Milne } \\
\text { Edwards and Haime, 1851) }\end{array}$ & 7 & - & V \\
\hline 2 & $\begin{array}{l}\text { Euphyllia glabrescens (Chamisso } \\
\text { and Eysenhardt, 1821) }\end{array}$ & 2 & - & NT \\
\hline
\end{tabular}


Table 3. Average coverage (\%), diversity and abundance patterns as well as index of diversity ( $\left.\mathrm{H}^{\prime}\right)$ and evenness (J') of Euphylliidae corals in Tioman Island

\begin{tabular}{|c|c|c|c|c|c|c|c|c|c|c|c|c|c|c|c|}
\hline \multirow[b]{2}{*}{ Genera } & \multirow[b]{2}{*}{ Species } & \multicolumn{13}{|c|}{ Tioman Reef Sites } & \multirow[b]{2}{*}{$\begin{array}{l}\text { Average } \\
\text { Coverage (\%) }\end{array}$} \\
\hline & & 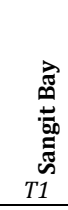 & 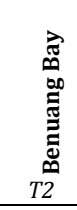 & 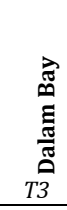 & 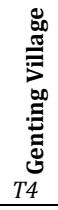 & 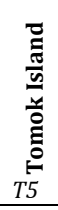 & 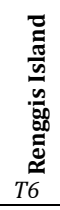 & 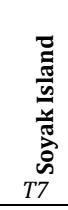 & 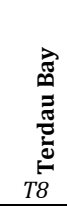 & 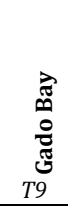 & 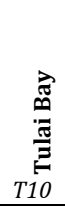 & 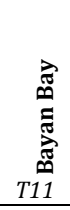 & 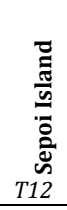 & 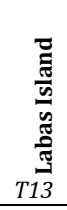 & \\
\hline Plerogyra & Plerogyra sinuosa & - & - & - & - & $\bullet$ & $\bullet$ & $\bullet$ & $\bullet \bullet$ & - & - & $\bullet$ & $\bullet \bullet$ & - & $19.1 \% \pm 0.7$ \\
\hline Physogyra & Physogyra lichtensteini & - & - & - & - & - & - & - & - & $\bullet$ & - & - & $\bullet$ & $\bullet$ & $6.9 \% \pm 0.4$ \\
\hline \multirow[t]{4}{*}{ Euphyllia } & Euphyllia ancora & $\bullet$ & $\bullet \bullet \bullet \bullet$ & $\bullet$ & - & - & - & - & $\bullet$ & $\bullet$ & - & $\bullet$ & $\bullet$ & $\bullet$ & $29.5 \% \pm 1.5$ \\
\hline & Euphyllia cristata & $\bullet$ & $\bullet \bullet$ & $\bullet \bullet$ & - & - & - & - & $\bullet$ & - & $\bullet$ & $\bullet$ & - & $\bullet$ & $17.9 \% \pm 1.0$ \\
\hline & Euphyllia divisa & $\bullet$ & $\bullet \bullet$ & $\bullet \bullet$ & - & - & - & - & $\bullet$ & $\bullet$ & - & $\bullet$ & - & $\bullet$ & $19.7 \% \pm 1.3$ \\
\hline & Euphyllia glabrescens & $\bullet$ & - & - & - & - & - & $\bullet$ & - & - & $\bullet$ & $\bullet$ & - & $\bullet$ & $6.9 \% \pm 0.4$ \\
\hline \multicolumn{2}{|c|}{ Total no. of species per site } & 4 & 5 & 3 & 0 & 1 & 1 & 3 & 4 & 4 & 2 & 6 & 3 & 6 & \\
\hline \multicolumn{2}{|c|}{ Total no. of colony per site } & 13 & 57 & 13 & - & 2 & 1 & 6 & 17 & 13 & 7 & 34 & 8 & 13 & \\
\hline \multicolumn{2}{|c|}{ Diversity index $\left(\mathrm{H}^{\prime}\right)$ per site } & 1.38 & 1.42 & 0.69 & - & - & - & 0.87 & 1.14 & 1.27 & 0.60 & 1.43 & 0.74 & 1.26 & $\sum H^{\prime}: 1.67$ \\
\hline \multicolumn{2}{|c|}{ Evenness index (J') per site } & 1.00 & 0.89 & 1.00 & - & - & - & 0.79 & 0.82 & 0.89 & 0.87 & 0.80 & 0.93 & 0.78 & $\sum J^{\prime}: 0.93$ \\
\hline
\end{tabular}

Note. $\bullet$ : one or few (1-5) $\quad \bullet \bullet:$ uncommon $(6-10) \quad \bullet \bullet:$ : common $(11-20) \quad \bullet \bullet \bullet:$ abundant $(21-30) \quad \cdots \bullet \bullet:$ dominant $(>30)$

Table 4. Average coverage (\%), diversity and abundance patterns as well as index of diversity ( $\left.\mathrm{H}^{\prime}\right)$ and evenness (J') of Euphylliidae corals in Redang Island

\begin{tabular}{|c|c|c|c|c|c|c|c|c|c|c|c|c|c|c|c|c|c|c|}
\hline \multirow[b]{2}{*}{ Genera } & \multirow[b]{2}{*}{ Species } & \multicolumn{16}{|c|}{ Redang Reef Site } & \multirow[b]{2}{*}{$\begin{array}{l}\text { Average } \\
\text { Coverage } \\
(\%)\end{array}$} \\
\hline & & R1 & R2 & R3 & 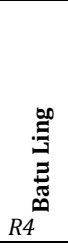 & 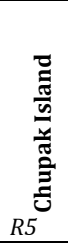 & 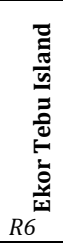 & 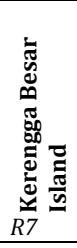 & 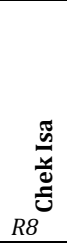 & 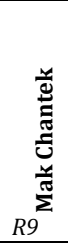 & 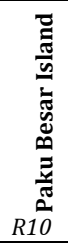 & 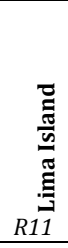 & 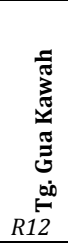 & 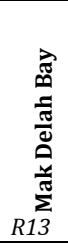 & 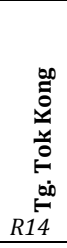 & 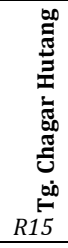 & 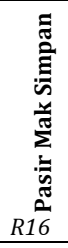 & \\
\hline Plerogyra & Plerogyra sinuosa & 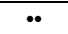 & - & $\cdots$ & $\ldots$ & m.. & - & 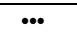 & ..... & 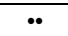 & 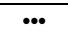 & 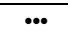 & 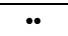 & 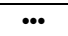 & ..... & 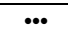 & ..... & $92.3 \% \pm 4.2$ \\
\hline \multirow[t]{4}{*}{ Euphyllia } & Euphyllia ancora & - & - & - & - & - & - & - & - & - & - & - & - & - & - & - & - & $0.3 \% \pm 0.1$ \\
\hline & Euphyllia cristata & $\bullet$ & - & - & - & - & - & - & - & - & - & - & - & - & - & - & - & $0.3 \% \pm 0.1$ \\
\hline & Euphyllia divisa & - & - & - & $\bullet$ & $\bullet$ & - & $\bullet$ & $\bullet$ & $\bullet$ & $\bullet$ & - & - & $\bullet$ & $\bullet$ & - & - & $5.9 \% \pm 0.4$ \\
\hline & $\begin{array}{l}\text { Euphyllia } \\
\text { glabrescens }\end{array}$ & - & - & - & - & $\bullet$ & $\bullet$ & - & - & - & - & - & - & - & - & - & - & $1.2 \% \pm 0.2$ \\
\hline \multicolumn{2}{|c|}{ Total no. of species per site } & 2 & 1 & 1 & 3 & 3 & 1 & 2 & 2 & 2 & 2 & 1 & 1 & 2 & 2 & 1 & 1 & \\
\hline \multicolumn{2}{|c|}{ Total no. of colony per site } & 11 & 5 & 16 & 70 & 30 & 3 & 17 & 33 & 11 & 15 & 14 & 7 & 18 & 36 & 13 & 40 & \\
\hline \multicolumn{2}{|c|}{ Diversity index $\left(\mathrm{H}^{\prime}\right)$ per site } & 0.30 & - & - & 0.39 & 0.20 & - & 0.36 & 0.14 & 0.30 & 0.58 & - & - & 0.59 & 0.29 & - & - & $\sum H^{\prime}: 0.33$ \\
\hline \multicolumn{2}{|c|}{ Evenness index (J') per site } & 0.88 & - & - & 0.35 & 0.18 & - & 0.52 & 0.20 & 0.43 & 0.84 & - & - & 0.85 & 0.42 & - & - & $\sum J^{\prime}: 0.21$ \\
\hline
\end{tabular}

Table 5. Average coverage (\%), diversity and abundance patterns as well as index of diversity ( $\left.\mathrm{H}^{\prime}\right)$ and evenness (J') of Euphylliidae corals in Payar Island

\begin{tabular}{|c|c|c|c|c|c|c|c|c|c|}
\hline \multirow{3}{*}{ Genera } & \multirow{3}{*}{ Species } & \multicolumn{7}{|l|}{ Payar Reef Site } & \multirow{3}{*}{$\begin{array}{l}\text { Average } \\
\text { Coverage } \\
(\%)\end{array}$} \\
\hline & & Banana Reef & Coral Garden & Kaca Island & Lembu Island & Segantang Bay & Anemone Garden & Tyre Reef & \\
\hline & & $P 1$ & $P 2$ & P3 & $P 4$ & P5 & $P 6$ & P7 & \\
\hline Physogyra & Physogyra lichtensteini & $\bullet \bullet \bullet \bullet$ & $\bullet \bullet \bullet \bullet$ & $\bullet$ & $\bullet \bullet$ & $\bullet$ & $\bullet \bullet$ & $\bullet$ & $\begin{array}{l}98.0 \% \pm \\
6.7\end{array}$ \\
\hline Euphyllia & Euphyllia glabrescens & $\bullet$ & - & - & - & - & - & - & $\begin{array}{c}2.0 \% \pm \\
0.3\end{array}$ \\
\hline \multicolumn{2}{|c|}{ Total no. of species per site } & 2 & 2 & 1 & 1 & 1 & 1 & 1 & \\
\hline \multicolumn{2}{|c|}{ Total no. of colony per site } & 56 & 36 & 10 & 18 & 7 & 15 & 6 & \\
\hline \multicolumn{2}{|c|}{ Diversity index $\left(\mathrm{H}^{\prime}\right)$ per site } & 0.09 & 0.21 & - & - & - & - & - & $\sum H^{\prime}: 0.10$ \\
\hline \multicolumn{2}{|c|}{ Evenness index (J') per site } & 0.13 & 0.30 & - & - & - & - & - & $\sum J^{\prime}: 0.14$ \\
\hline
\end{tabular}

In terms of diversity and evenness the value of $\mathrm{H}^{\prime}$ and J' of euphylliids did not differ significantly ( $p>0.05)$ between reef sites in Tioman, Redang and Payar Islands. However, total average values of $\mathrm{H}^{\prime}$ and J' of euphylliids differed significantly between study areas in Tioman, Redang and Payar islands. Tioman showed higher total index of diversity and evenness indices ( $\left.\mathrm{H}^{\prime}: 1.67, \mathrm{~J}^{\prime}: 0.93\right)$ compared to Redang ( $\left.H^{\prime}: 0.33, J^{\prime}: 0.21\right)$ and Payar Islands (H': 0.10, J':0.14).

\section{Discussion}

Present results indicate differences in the average percentage coverage, diversity and abundance of the Euphylliidae corals in the study areas. These corals were found to be more diversified and abundant in Tioman compared to Redang and Payar. In terms of the average coverage of euphylliids, E. ancora was found to be the most 
dominant species which contributed the highest percentage in Tioman while P. sinuosa in Redang and P. lichtensteini in Payar. These differences can be attributed to the differences in reef morphology and substrate condition between study areas. Furthermore, the ability of an individual species to tolerate the changes in environmental parameters or anthropogenic disturbances are the other factors that determine the species occurrence in a particular reef area (Veron et al., 2011; Hughes et al., 2012; Hennige et al., 2013; Williams et al., 2013).

In Tioman Island, the distribution of Euphylliidae corals is scattered except at T4, where euphylliids were not recorded. Among euphylliid species, E. ancora, E. divisa and $E$. cristata ranged from common to dominant at T2 (east coast area) compared to other reef sites. The highest diversity of euphylliid species was recorded at T11 and T13 (remote reef area) and the lowest diversity was seen at T4, $\mathrm{T} 5$ and $\mathrm{T} 6$ (west coast area). The west coast area of Tioman is highly exposed to human impacts since there are many chalets and resorts along the seafront. Moreover, this area is also popular for water sport activities such as snorkeling, diving and boating. The extensive coastal development and widespread tourism related activities have contributed to many negative impacts on coral reefs which could be responsible for reduced species diversity (Harborne et al., 2000; Toda et al., 2007; Shahbudin et al., 2017).

In comparison to Tioman, the diversity of euphylliids was slightly lower in Redang. However, total number of species recorded per site in Redang was relatively low compared to Tioman. This could be due to low habitat heterogeneity in this island. This is consistent with the previous study that indicated that both the islands resembled in many geomorphological features - shallow depth and fringing reefs, but differed in habitat heterogeneity (Harborne et al., 2000). Habitat heterogeneity has been reported to be among the important factors that influence the diversity and abundance of corals. Habitat diversity provides many micro- and macro-habitats and niches that can support more species in an area (Planes et al., 2012; Hennige et al., 2013). In terms of species occurrence, $P$. sinuosa was dominant in Redang, covering $92.3 \%$ in average. This species existed in small solitary and large colonial forms, commonly in protected reef environments that are sheltered from strong waves and currents (Veron, 2000). The solitary form of this species was mostly found attached to the crevices and hangover to the rocky substrates while the colonial form to the bottom substrates (Veron, 2000). The reef slope morphology in Redang which was dominated by patches of boulders, and rocky and sandy substrates provided the habitat preferences for P. sinuosa to grow and propagate.

$P$. lichtensteini which was a dominant species in Payar, with a $98.0 \%$ coverage, is known for tolerating stress and is able to survive in turbid water and muddy substrate conditions (Chua and Charles, 1980; Veron, 2000) as well as in the environment where penetration of light is low (McClanahan et al., 2007) due to heavy sedimentation and siltation (Chua and Ross, 2000; Jonsson, 2003; Toda et al.,
2007). It has been reported that large amounts of suspended sediments have been transported by many rivers along the east coast of Sumatra and the west coast of Peninsular Malaysia (Lim, 1998; Soegiarto, 2000), leading to deterioration of the environmental conditions and stress on marine life. Other studies have also indicated that the concentrations of suspended sediments including silt and other particulate matters along the west coast were higher ( $49.8 \mathrm{mg} / \mathrm{cm} 2 /$ day at depth ranged $1-3 \mathrm{~m}$ ) compared to the east coast of Peninsular Malaysia $(3.5 \mathrm{mg} / \mathrm{cm} 2 /$ day at a depth of 1 - $12 \mathrm{~m}$ ) (Lee and Mohamed, 2011). Apart from that, the colony growth of this species in the form of massive and platy structures may account for higher tolerance to resist strong wave and current actions compared to branching coral corals (Kenyon et al., 2006; McClanahan et al., 2007). In the light of these findings it can be postulated that a multiplicity of factors is responsible for dominance of the coral species surrounding reef sites in Payar.

\section{Conclusion}

In conclusion, 671 euphylliids belonging to 6 species and 3 genera were recorded in the study area. E. ancora was found to be the most dominant species in Tioman, $P$. sinuosa in Redang and P. lichtensteini in Payar. Research finding indicated that the diversity and evenness index were higher in Tioman compared to Redang and Payar. The diversity and abundance of the Euphylliidae corals are influenced by various factors such as reef morphology and substratum condition. Moreover, human activities may also contribute to reducing coral diversity, especially those belonging to the endangered categories. To protect the coral reef ecosystems the unsustainable coastal developments and other human activities should be monitored and controlled through enforcement regulations. The information generated by this study provides useful data to the Department of Marine Park Malaysia and other institutions about the occurrence of near threatened and vulnerable euphylliid species in Peninsular Malaysia for a better coral reefs management plan.

\section{Acknowledgements}

This research was supported by E-Science fund (SF-12-0160045) under the Ministry of Science, Technology and Innovation, Malaysia (MOSTI). The authors would like to express their gratitude to the Sea-Quest team of Tropical Marine Ecology Laboratory, IIUM for their invaluable assistance throughout the sampling period. Appreciation also goes to the Department of Marine Park Malaysia for providing a permit for this study in marine protected areas of Tioman, Redang and Payar Islands.

\section{References}

Affendi, Y. A. \& Rosman, F.R. (2012). Current knowledge on sclerectinian coral diversity of Peninsular Malaysia. In: Malaysia's Marine Biodiversity: Inventory and Current Status. (I. Kamarruddin, C.A.R. Mohamed, M.J. Rozaimi, et al. eds.), pp 21-31. Department of Marine Park Malaysia, Putrajaya, Malaysia 
Benzoni, F., Arrigoni, R., Waheed, Z. et al. (2014). Phylogenetic relationships and revision of the genus Blastomussa (Cnidaria: Anthozoa: Scleractinia) with description of a new species. Raffles Bulletin of Zoology 62, 358-378.

Carpenter, K.E., Abrar, M., Aeby, G. et al. (2008). One-third of reefbuilding corals face elevated extinction risk from climate change and local impacts. Science 321, 560-563.

Chua, T.E. \& Charles, J.J.C. (1980). Coastal resources of East Coast Peninsular Malaysia. In: An Assessment in Relation to Potential Oil Spills. Pp 507, Universiti Sains Malaysia, Penang, Malaysia

Chua, T.E. \& Ross, S.A. (2002). Creating a shared vision for environmental management in the Straits of Malacca. In: Tropical Marine Environment: Charting Strategies for the Millennium. (F.M. Yusoff, M. Shariff, H.M. Ibrahim. Et al. eds.), pp 19-33. Malacca Straits Research and Development Centre (MASDEC), Universiti Putra Malaysia, Serdang, Malaysia

Department of Marine Park Malaysia (2013). Ministry of Natural Resources and Environment, Putrajaya, Malaysia. Retrieved from http://www.dmpm.nre.gov.my

Fukami, H., Chen, C.A., Budd, A.F. et al. (2008). Mitochondrial and nuclear genes suggest that stony corals are monophyletic but most families of stony corals are not (Order Scleractinia, Class Anthozoa, Phylum Cnidaria). PloS ONE 3, e3222.

Hammer, Ø., Harper, D.A.T. \& Ryan, P.D. (2001). PAST: Paleontological statistics software package for education and data analysis. Palaeontologia Electronica 4, 9.

Harborne, A., Fenner, D., Barnes, A. et al. (2000). Status report on the coral reefs of the East Coast of Peninsula Malaysia. Technical Report. Department of Fisheries Malaysia, Kuala Lumpur, Malaysia

Hennige, S.J., Suggett, D.J., Hepburn, L. et al. (2013). Coral reefs of the Wakatobi: processes of reef growth and loss. In: Marine Research and Conservation in the Coral Triangle: the Wakatobi Marine National Park (J. Clifton, R.K.F. Unsworth \& D.J. Smith, eds.), pp 27-44. Nova Science.

Hughes, T.P., Baird, A.H., Dinsdale, E.A. et al. (2012) Assembly rules of reef corals are flexible along a steep climatic gradient. Current. Biology 22, 736-741.

Jonsson, D. (2003). An inventory of coral reefs in the Langkawi archipelago, Malaysia: assessment and impact study of sedimentation, Undergraduate Thesis. Department of Animal Ecology, Uppsala University, Sweden

Kenyon, J.C., Vroom, P.S., Page, K.N. et al. (2006). Community structure of hermatypic corals at French Frigate Shoals, Northwestern Hawaiian Islands: Capacity for resistance and resilience to selective stressors 1. Pacific Science 60, 153-175.

Kitahara, M.V., Cairns, S.D., Stolarski, J. et al. (2010). A comprehensive phylogenetic analysis of the Scleractinia (Cnidaria, Anthozoa) based on mitochondrial C01 sequence data. PloS ONE 5, e11490.

Khodzori, M.F.A., Saad, S., Nordin, N.F.H. et al. (2015). Diversity and distribution of Euphylliidae corals in Tioman Island: emphasis on the genetic variation of Euphyllia cristata. Jurnal Teknologi 77, 17-22.

Khodzori, F.A., Saad, S., Nordin, N.F.H. et al. (2017). Taxonomic status of Euphylliidae corals in Peninsular Malaysia based on morphological structures and phylogenetic analyses using mitochondrial COI gene. Russian Journal of Marine Biology 43, 118-126.

Lee, J.N. \& Mohamed, C.A.R (2011). Accumulation of settling particles in some coral reef areas of Peninsular Malaysia. Sains Malaysiana 40, 549554.

Liew, H.C., Hii, Y.S., Bachok, Z. et al. (2012). A Guide to Collecting Digital Videos for Coral Reef Surveys and Monitoring Purposes. 28 pp. Department of Marine Parks Malaysia

Lim, L.C. (1998). Carrying Capacity Assessment of Pulau Payar Marine Park, Malaysia-Bay of Bengal Programme. Technical Report. BOBP/REP/79. Bay of Bangal Programme, Madras, India
McClanahan, T.R., Ateweberhan, M., Graham, N.A.J. et al. (2007). Western Indian Ocean coral communities: bleaching responses and susceptibility to extinction. Marine Ecology Progress Series 337, 1-13.

Pielou, E.C. (1966). The measurement of diversity in view publication stats different types of biological collections. Journal of Theoretical Biology 13, 131-144.

Planes, S., Lecchini, D., Mellin, C. et al. (2012). Environmental determinants of coral reef fish diversity across several French Polynesian atolls. Comtes Rendus Biologies 335, 417-423.

Rhyne, A.L., Tlusty, M.F. \& Kaufman, L. (2014). Is sustainable exploitation of coral reefs possible? A view from the standpoint of the marine aquarium trade. Current Opinion Environment Sustainability 7, 101-107.

Shahbudin, S., Akmal, K.F., Faris, S. et al. (2017). Current status of coral reefs in Tioman Island, Peninsular Malaysia. Turkish Journal of Zoology 41, 294-305.

Shannon, C. E. \& Weaver, W. (1949). The Mathematical Theory of Communication. University of Illinois press

Soegiarto, A. (2000). Pollution management and mitigation in the Straits of Malacca: priorities, uncertainties and decision making. In: Towards Sustainable Management of the Straits of Malacca (M. Shariff, F.M. Yusoff, N. Gopinath. et al. eds.) pp 503-518, Malacca Straits Research and Development Center (MASDEC), Faculty of Science and Environmental Studies, Universiti Putra Malaysia, Serdang, Malaysia

Thornhill, D.J. (2012). Ecological Impacts and Practices of the Coral Reef Wildlife Trade. Defenders of Wildlife, Washington

Tissot, B.N., Best, B.A., Borneman, E.H. et al. (2010). How US ocean policy and market power can reform the coral reef wildlife trade. Marine Policy 34, 1385-1388.

Toda, T., Okashita, T., Maekawa, T. et al. (2007). Community structures of coral reefs around Peninsula Malaysia. Journal of Oceanography 63 113-123.

Veron, J.E.N. (2000). Corals of the World. Volumes 1-3. 68 pp. Australian Institute of Marine Science, Townsville, Australia

Veron, J.E.N., DeVantier, L.M., Turak, E. et al. (2011). The coral triangle. In: Coral Reefs: An Ecosystem in Transition. pp 47-55. Springer, Netherlands

Wabnitz, C., Taylor, M., Green, E. et al. (2003). From Ocean to Aquarium: The Global Trade in Marine Ornamental Species. 64 pp. UNEP-WCMC, Cambridge. United Kingdom

Waheed, Z. \& Hoeksema, B.W. (2013). A tale of two winds: species richness patterns of reef corals around the Semporna peninsula, Malaysia. Marine Biodiversity 43, 37-51.

Waheed, Z. \& Hoeksema, B.W. (2014). Diversity patterns of scleractinian corals at Kota Kinabalu, Malaysia, in relation to exposure and depth. Raffles Bulletin of Zoology 62, 66-82.

Waheed, Z., Harald, G.J., Mil, V. et al. (2015). Coral reefs at the northernmost tip of Borneo: an assessment of scleractinian species richness patterns and benthic reef assemblages. PLoS ONE 10, e0146006

Wilkinson, C. (2008). Status of Coral Reefs of the World: 2008. 296 pp. Global Coral Reef Monitoring Network and Reef and Rainforest Research Center. Townsville, Australia

Williams, G.J., Smith, J.E., Conklin, E.J. et al, (2013). Benthic communities at two remote Pacific coral reefs: effects of reef habitat, depth, and wave energy gradients on spatial patterns. PeerJ 1, e81.Anthony, P.D. \& Hawkins, A.D. (1983). Spectral sensitivity of the cod, Gadus morphua L. Marine Behaviour and Physiology 10, 145-166. 